\title{
Los médicos que realizan su labor asistencial e investigadora en unidades de dolor en el uso de internet: identificación, caracterización y determinantes
}

\author{
The medical doctors who perform their care and research \\ work in pain units in the use of the internet: identification, \\ characterization and determinants
}

J. Muriel Fernández, M. B. García-Cenador, N. López-Valverde, C. Muriel Villoria y M. J. Sánchez-Ledesma Departamento de Cirugía. Universidad de Salamanca, España

\section{ABSTRACT}

Objectives: The information provided by the Labor Conditions and Health Surveys (ECTS) allows for the formulation of public action programs that generate changes, set priorities and evaluate trends. Since 1987, when the first ECTS was carried out in Spain, this instrument has been consolidated as a data source for the information and communication technology system. The results show the need to establish the advance of the technological innovations in health and biomedical research. These advances will be decisive in the improvement of health, since they will involve new forms of medical care that contribute to the increase of life expectancy, the reduction of pain and the management of diseases. The objective of this study is to assess the level of acceptance of new technologies in physicians who treat pain through specific units.

Matherial and method: A study was carried out to verify the use of ICTs in doctors who perform their care and research work in Pain Units. This study consists of an online survey, sent via email to the students of the Master's Degree in Pain Treatment at the University of Salamanca. After the systematic collection of the information, the statistical analysis was carried out, addressing the frequency of the variables and the statistically significant relationships relevant to our study. Subsequently, a factor analysis, a cluster analysis and new composite variables are constructed to synthesize

Muriel Fernández J, García-Cenador MB, López-Valverde N, Muriel Villoria C, Sánchez-Ledesma MJ. Los médicos que realizan su labor asistencial e investigadora en unidades de dolor en el uso de internet: identificación, caracterización y determinantes. Rev Soc Esp Dolor 2019;26(5):284-292

\section{RESUMEN}

Objetivos: La información proporcionada por las Encuestas de Condiciones de Trabajo y Salud (ECTS) permite la formulación de programas de acción públicos que generen cambios, fijen prioridades y evalúen tendencias. Desde 1987 que se realizó la primera ECTS en España, este instrumento se ha ido consolidando como fuente de datos del sistema de las tecnologías de la información y la comunicación. Los resultados muestran la necesidad de establecer el avance de las innovaciones tecnológicas sanitarias y la investigación biomédica. Estos avances serán determinantes en la mejora de la salud, ya que supondrán nuevas formas de cuidados médicos que contribuyen al aumento de la esperanza de vida, la reducción del dolor y la gestión de las enfermedades. El objetivo de este estudio es valorar el nivel de aceptación de las nuevas tecnologías en médicos que traten el dolor a través de unidades específicas.

Material y métodos: Se realizó un estudio para comprobar el uso de las TIC en médicos que realizan su labor asistencial y de investigación en Unidades de Dolor. Este estudio consta de una encuesta online, enviada a través de correo electrónico a los alumnos del máster en Tratamiento del Dolor de la Universidad de Salamanca. Tras la recogida sistemática de la información, se realizó el análisis estadístico abordando la frecuencia de las variables y las relaciones estadísticamente significativas relevantes para nuestro estudio.

Recibido 19-10-2018

Aceptado 01-07-2019

Correspondencia: Jorge Muriel Fernández murimuriel@gmail.com 
the data, to which, finally, an analysis was made using a logistic regression methodology through the Binomial Logit model. Results: Of the medical professionals who answered our survey, it was obtained that those who are men and work in Pain Units were $39.46 \%$ and in Palliative Care they were $21.35 \%$. Adding this data, we obtain that $75.94 \%$ of professionals work in public entities, $9.72 \%$ in private entities and $11.05 \%$ in both. More than $55 \%$ of the professionals surveyed "never use computers or Information and Communication Technologies for the remote realization of diagnostic evaluation or prescription of therapeutic measures".

Conclutions: The results obtained show that Pain Units physicians consider the applications of Information and Communication Technologies to be a relevant element for clinical practice. In addition, given the clinical experience of professionals, the need for organizational changes associated with new computer systems is confirmed.

Key words: Information technologies, pain units, care and research work, internet.
Posteriormente se realizó un análisis utilizando una metodología de regresión logística a través del modelo Logit binomial.

Resultados: De los profesionales médicos que contestaron a nuestra encuesta, se obtuvo que aquellos que son varones y trabajan en Unidades del Dolor eran un $39,46 \%$ y en Cuidados Paliativos eran un 21,35 \%. Agregando estos datos obtenemos que el 75,94\% de los profesionales trabajan en entidades públicas, el $9,72 \%$ en entidades privadas y el 11,05 \% en ambas. Más del $55 \%$ de los profesionales encuestados "Nunca utilizan ordenadores o Tecnologías de la Información y la Comunicación para la realización remota de evaluación diagnóstica o prescripción de medidas terapéuticas".

Conclusiones: Los resultados obtenidos nos muestran que los médicos de unidades de dolor consideran un elemento relevante las aplicaciones de las Tecnologías de la Información y la Comunicación para la práctica clínica. Además, se constata, dada la experiencia clínica de los profesionales, la necesidad de cambios organizativos asociados a los nuevos sistemas informáticos.

Palabras clave: Tecnologías de la información, unidades de dolor, labor asistencial e investigadora, internet.

\section{INTRODUCCIÓN}

Las encuestas forman parte de los sistemas de información en salud y tienen como objetivo específico aportar información sobre la percepción de los médicos con respecto a las aplicaciones de las Tecnologías de la Información y la Comunicación (TIC). Información que, junto con otras fuentes, permite la formulación de programas de acción públicos que generen cambios, fijen prioridades y evalúen tendencias (1).

Las primeras encuestas datan de los años 70, y desde entonces se han extendido ampliamente en los países más industrializados.

Entre las ventajas de las encuestas, se ha señalado el hecho de que constituyen un procedimiento relativamente rápido, de bajo coste, para conocer los factores relacionados con el trabajo, en un momento y territorio determinado. Además, los contenidos de las encuestas son flexibles y se pueden adaptar a los temas de mayor interés en cada momento. Por último, las encuestas permiten, si los métodos y las cuestiones son similares, realizar comparaciones a través de territorios y a través del tiempo. Por contra, entre las desventajas hay que destacar la naturaleza indirecta de la información que se recoge (basada en la percepción en este caso del alumno], la imprecisión de las formulaciones que puede hacer que sean entendidas de manera diferente por diferentes encuestados o la diferente percepción de cada uno de los encuestados.

En los inicios de la década de 1990, con la introducción de nuevas facilidades de interconexión y herramientas gráficas simples para el uso de la red, se inició el auge que actualmente le conocemos a internet. Este crecimiento masivo trajo consigo el surgimiento de un nuevo perfil de usuarios, en su mayoría de personas comunes no ligadas a los sectores académicos, científicos y gubernamentales [1].

La revolución de las TIC es la base sobre la que se ha desarrollado el avance de las innovaciones tecnológicas sanitarias y la investigación biomédica. Estos avances han sido determinantes en la mejora de la salud, ya que han supuesto nuevas formas de cuidados médicos que contribuyen al aumento de la esperanza de vida, la reducción del dolor y la gestión de las enfermedades (1).

La Organisation for Economic Co-operation and Development (OECD) (2) también destaca la potencialidad de las TIC para la mejora en la actuación de los sistemas de salud. Anderson y cols. [3] sugieren que la adopción de estas tecnologías podría facilitar la disminución del gasto y el aumento de la calidad de los servicios.

Como espacio de comunicación, internet podría transformar los procesos de interacción entre los diferentes agentes del sistema sanitario y entre estos y las organizaciones donde desarrollan su actividad. Todo ello facilitaría el aumento de los flujos de información, mejoraría la accesibilidad al sistema y podría generar comunidades en red de apoyo mutuo [4].

El presente trabajo tiene como objetivo principal valorar el nivel de aceptación de las nuevas tecnologías en médicos que traten el dolor a través de unidades específicas (Unidades del Dolor), utilizando para ello los resultados obtenidos tras aplicar una encuesta a un grupo de médicos inscritos en el Máster en Tratamiento del Dolor de la Universidad de Salamanca.

\section{MATERIAL Y MÉTODOS}

El corpus analizado se obtuvo a partir de la elaboración de un cuestionario online que fue enviado a través 
del correo electrónico. En este estudio participaron los 531 alumnos inscritos en el Máster en Tratamiento del Dolor a lo largo de sus catorce ediciones.

Para superar las cuestiones relacionadas con la privacidad y la confidencialidad de la encuesta, el lanzamiento de los correos electrónicos fue realizado por la administración del Máster, mientras que las respuestas obtenidas eran almacenadas en los servidores de la Cátedra Extraordinaria del Dolor de la Universidad de Salamanca.

Los correos electrónicos constaban de un texto en el que se incluía una explicación del proyecto de investigación, una petición de colaboración y un enlace al cuestionario.

Cada correo electrónico contenía un enlace a la encuesta, lo que garantiza la unicidad de respuesta. La herramienta de encuestas online está diseñada para trabajar a través de la web, de manera que tanto la recogida de datos como su posterior descarga se hace de manera online.

Desde un punto de vista metodológico, en nuestro estudio podemos identificar tres etapas: el diseño de los instrumentos, la recogida sistemática de la información y el análisis estadístico realizado. Así, en función de las preguntas de investigación propuestas, las hipótesis planteadas y los objetos de estudio seleccionados, hemos diseñado el cuestionario, así como el estudio estadístico a realizar.

De esta forma se ha construido un cuestionario teniendo en cuenta las características del medio a utilizar (internet), que nos ha permitido una recogida sistemática de la información, facilitando la identificación de los items seleccionados en la encuesta.

Posteriormente hemos realizado el análisis estadístico siguiendo el esquema que exponemos a continuación. En primer lugar, abordamos la frecuencia de las variables y las relaciones estadísticamente significativas relevantes para nuestro estudio. En segundo lugar, mediante análisis de factores, análisis de clúster y la construcción de nuevas variables compuestas, hemos sintetizado los datos. Esta reducción de la información nos ha permitido la identificación de los individuos en grupos homogéneos internamente y heterogéneos externamente. Tras esta identificación, el análisis de las relaciones estadísticamente significativas nos ha llevado a caracterizar a estos individuos. Finalmente, hemos realizado un análisis causal que nos permite estimar la presencia o ausencia de una característica según los valores de un conjunto de variables predictoras o independientes que se asocian estadísticamente. Para realizar este tipo de análisis hemos utilizado una metodología de regresión logística a través del modelo Logit binomial.

Además de estas cuestiones, antes de abordar las metodologías de cada uno de los estudios realizados, presentamos algunas consideraciones generales sobre la aplicación Survey Manager, de Netquest, la herramienta de encuestas online que hemos utilizado. Esta herramienta está diseñada para trabajar a través de la web. La encuesta consta de 21 ítems organizados alrededor de varios tipos de preguntas: aquellas de índole general, como el sexo o la edad, el lugar de trabajo, la especialidad médica, otras como el uso de internet en su práctica clínica o el uso de la TIC, o sobre la relación médico-paciente.

\section{RESULTADOS}

El número de respuestas obtenidas ha sido del 73,25 \% (389 respuestas), siendo no válidas 19 de ellas: 12 por contestación de forma incorrecta, 4 por no estar de acuerdo con el cuestionario y 3 por pedir una compensación económica, siendo los cuestionarios válidos para realizar el estudio 370. El trabajo de campo se realizó durante los meses de julio a septiembre del 2017

La distribución de los porcentajes y las combinaciones reflejan la diversidad en los servicios de los distintos sistemas de salud. No obstante, podemos destacar la importancia de los centros de titularidad y gestión pública, Unidad del Dolor [39,46 \%), Cuidados Paliativos [21,35\%) y en los Centros de Atención Primaria (CAP); agregando estos datos obtenemos que el 75,94\% de los profesionales trabajan en entidades públicas, el $9,72 \%$ en entidades privadas y el 11,05 \% en ambas.

Si abordamos solo aquellos profesionales que han declarado que desarrollan su actividad en un único lugar, observamos que el $60,81 \%$ lo hacen en un Hospital y el 15,13\% en un Centro de Atención Primaria.

Existe un comportamiento diferencial que nos muestra que son los profesionales con mayor edad los que en un porcentaje mayor desarrollan su actividad profesional en diferentes lugares de trabajo, mientras que los profesionales más jóvenes desarrollan en un porcentaje mayor su actividad en un único lugar de trabajo.

Otra cuestión importante que caracteriza a los profesionales médicos es su especialidad, es decir, el área de conocimiento de la medicina que abordan en su práctica profesional. El nacimiento de nuevas especialidades (dolor y cuidados paliativos) está asociado con el desarrollo de la medicina como ciencia y la necesidad de acotar y profundizar en aspectos determinados de la salud en el paciente crónico. El 68,1 \% de los médicos encuestados pertenecen a especialidades médicas que no realizan intervenciones quirúrgicas. Dentro de estas, destacan la especialidad de Anestesiología [34,5\%), Medicina Familiar y Comunitaria $(7,8 \%$ ) y Medicina Interna (6,6\%). Un 22,1 \% de los profesionales señalan que pertenecen a especialidades que abordan cuestiones transversales a las especialidades quirúrgicas y médicas.

Por último, además de la especialidad, hemos abordado las tareas que realizan los profesionales médicos en su actividad profesional. El 53,6\% de los encuestados realizan únicamente tareas relacionadas con la práctica asistencial en el tratamiento del dolor, porcentaje que aumenta hasta el 90,2\% en el caso de combinar la actividad asistencial con otras tareas (por ejemplo, realizar anestesia y tratamiento del dolor]. Tan solo un 4,9\% de los profesionales médicos se dedican exclusivamente a la Planificación, Dirección o Gestión en las Unidades del Dolor. Este porcentaje disminuye a menos de un $3 \%$ en los casos de dedicarse con exclusividad a la investigación o a la docencia.

Hemos abordado la caracterización de prácticas avanzadas en el uso de internet mediante el resumen de la información contenida en los principales grupos de variables relacionadas por un lado con los médicos que realizan su labor asistencial e investigadora en Unidades del dolor y, por otro lado, el resto. 
Aquellos profesionales caracterizados por este tipo de prácticas los denominaremos "Médicos en dolor en el uso sistemas de información".

Para sintetizar empíricamente los factores relacionados con los Médicos en Dolor en el uso de internet hemos procedido a realizar análisis clúster no jerárquicos (Tabla I) con el objetivo de profundizar en el conocimiento del perfil del profesional médico en función de su similitud con el grado de adopción de estas prácticas avanzadas. Este análisis se ha realizado tomando como

TABLA I

UNA CARACTERIZACIÓN DE LAS PRÁCTICAS AVANZADAS EN EL USO DE INTENSIVOS DE INTERNET DE LOS PROFESIONALES MÉDICOS DE UNIDADES DE DOLOR (MÉTODO DE ANÁLISIS: CLÚSTER NO JERÁRQUICOS; CENTROS DE LOS CONGLOMERADOS FINALES]

\begin{tabular}{|l|c|c|c|}
\hline \multicolumn{4}{|c|}{ CONGLOMERADOS } \\
\hline & $\begin{array}{c}1 \\
(n=132)\end{array}$ & $\begin{array}{c}2 \\
(n=238)\end{array}$ & ANOVA \\
\hline $\begin{array}{l}\text { Orientados } \\
\text { información } \\
\text { internacional }\end{array}$ & 0,538 & $-0,399$ & $447,790^{\star}$ \\
\hline $\begin{array}{l}\text { Orientados } \\
\text { información } \\
\text { nacional }\end{array}$ & 0,265 & $-0,197$ & $87,390^{*}$ \\
\hline $\begin{array}{l}\text { Internet } \\
\text { orientados } \\
\text { información } \\
\text { investigación }\end{array}$ & 0,383 & $-0,265$ & $171,911^{\star}$ \\
\hline $\begin{array}{l}\text { Internet } \\
\text { orientados a } \\
\text { información } \\
\text { institucional }\end{array}$ & $-0,103$ & 0,055 & $9,693^{*}$ \\
\hline $\begin{array}{l}\text { Participa en } \\
\text { internet }\end{array}$ & 0,408 & $-0,257$ & $181,693^{*}$ \\
\hline $\begin{array}{l}\text { Produce } \\
\text { información } \\
\text { en internet }\end{array}$ & 0,599 & $-0,377$ & $452,151^{*}$ \\
\hline $\begin{array}{l}\text { Comunicar } \\
\text { con pacientes }\end{array}$ & 0,293 & $-0,184$ & $88,603^{*}$ \\
\hline
\end{tabular}

${ }^{\star} \mathrm{P}<0,001$

variables de clasificación el máximo número de factores, teniendo en cuenta la significatividad estadística adecuada y la mínima pérdida de casos.

A partir del establecimiento de los conglomerados se han definido dos tipologías o patrones de los profesionales médicos. Los conglomerados obtenidos pueden distinguirse en dos: un conglomerado con los médicos que no trabajan en Unidades de Dolor (grupo 1) y otro con médicos de Unidades de Dolor (grupo 2). El primer grupo son 132 médicos y el segundo grupo, 238 (Tabla II).

El primer grupo se caracteriza por el signo negativo, lo que puede interpretarse como un uso no intensivo de los sistemas de información. El segundo grupo destaca por el uso intensivo de los sistemas de información basados en las TIC. Para la interpretación de los conglomerados resultantes, se ha medido la asociación entre las variables de origen y los diferentes grupos, que nos ha permitido la atribución de significatividad estadística a las diferencias obtenidas.

Una vez definido el perfil de usuario intensivos en el uso de internet (médico en UD) y en el uso intensivo de los sistemas de información en la práctica clínica (médico unidad del dolor en el uso sistemas de información) hemos comprobado que existe una asociación significativa entre ambos indicadores. Esta asociación nos muestra un porcentaje mayor de lo esperado de médicos en red entre los médicos de unidad de dolor en el uso de sistemas de información.

Además de esta asociación con la finalidad de profundizar en las características del médico en UD, hemos abordado las asociaciones significativas entre este perfil y las diferentes dimensiones de nuestro estudio. Si abordamos las variables relacionadas con los aspectos sociodemográficos observamos que existe un porcentaje de médicos en UD mayor de lo esperado entre los hombres y entre los mayores de 41 años.

Si abordamos la dimensión relacionada con la actividad profesional, en primer lugar observamos que existe un porcentaje mayor de lo esperado de médicos en red que trabajan en más de un lugar cuya titularidad es mixta (pública-privada). De aquellos profesionales que trabajan en un único lugar, se constata un porcentaje mayor de lo esperado de médicos en UD en los hospitales.

En segundo lugar, observamos que existe un porcentaje mayor de lo esperado de médicos en UD entre aquellos profesionales que realizan más de dos

\section{TABLA II}

PERFIL DE USUARIO DE UNIDAD DE DOLOR EN EL USO DE INTERNET (MÉDICO EN RED) Y PERFIL DE USUARIO EN EL USO INTENSIVO DE LOS SISTEMAS DE INFORMACIÓN (MÉDICO UD EN EL USO SISTEMAS DE INFORMACIÓN]

(PORCENTAJE DE MÉDICOS Y SIGNIFICACIÓN ESTADÍSTICA CHI CUADRADO)

\begin{tabular}{|l|c|c|c|}
\hline & $\begin{array}{c}\text { Médico } \\
\text { en UD }\end{array}$ & $\begin{array}{c}\text { Médico } \\
\text { tradicional }\end{array}$ & $\begin{array}{c}\text { Significación } \\
\text { estadistica } p\end{array}$ \\
\hline $\begin{array}{l}\text { Médico } \\
\text { Unidad del } \\
\text { Dolor en } \\
\text { el uso de } \\
\text { sistemas de } \\
\text { información }\end{array}$ & 61,8 & 38,2 & 0,001 \\
\hline $\begin{array}{l}\text { Resto de } \\
\text { médicos }\end{array}$ & 38,4 & 61,6 & 0,001 \\
\hline
\end{tabular}


actividades: práctica asistencial, docencia e investigación y práctica asistencial, docencia, investigación y gestión.

Respecto a la dimensión relacionada con la intensidad, la experiencia y la utilidad en el uso de internet [Tabla III) se constata que existe un porcentaje mayor de lo esperado de médicos en UD entre aquellos que utilizan con mayor intensidad internet (todos los días), tanto fuera como dentro de su trabajo; entre aquellos que tienen una mayor experiencia en el uso de internet, ya que hace más tiempo que están conectados [antes del año 2000); y, por último, entre aquellos que consideran que internet tiene mayor utilidad.

Las consecuencias del uso de internet (Tabla IV) se constata en primer lugar que existe un porcentaje mayor de lo esperado de médicos en UD que valoran la información sobre salud disponible en internet como muy relevante. En segundo lugar, se observa un porcentaje mayor de lo esperado de médicos en UD que consideran que los pacientes recurran a la información sobre salud disponible en internet mejora mucho o mejora su calidad de vida y su autonomía. En tercer lugar, se constata un mayor porcentaje de este tipo de profesional entre aquellos que comparte o discuten información sobre salud que los pacientes consultan en internet y que consideran que la consulta de este medio mejora el conocimiento del paciente y facilita su tratamiento. Finalmente, existe también un porcentaje mayor de lo esperado de médicos en UD entre aquellos profesionales que declaran que el uso de internet en su actividad profesional mejora la comunicación con otros profesionales; mejora la eficiencia y la productividad de su trabajo; mejora tanto la atención como la comunicación con los pacientes.

En cuanto a la dimensión relacionada con la valoración de las TIC que realizan los profesionales encuestados (Tabla V) se observa que existe un porcentaje mayor de lo esperado de médicos en red que están totalmente de acuerdo con que la existencia de datos informatizados que permita ver la evolución del estado clínico del paciente es muy útil para la práctica clínica; con que el uso masivo de las Tecnologías de la Información y la Comunicación existe un mayor control de los errores y que es tan importante innovar en las cuestiones organizativas de los servicios y la institución como invertir en nuevas prácticas a través del uso de estas tecnologías.

Con el objetivo de determinar los factores que impulsan el uso intensivo de internet, hemos formulado un modelo en que la variable dependiente es el indicador del médico en red. En el modelo que queremos estimar, la variable dependiente es dicotómica, por lo que utilizaremos una metodología de regresión logística a través del modelo Logit binomial.

De los resultados de la estimación del modelo se desprenden las siguientes consideraciones. El porcentaje de observaciones clasificados correctamente es del 79,2 \% (el 68,4\% de médicos en red y el 86,6 de médicos tradicionales]. Con respecto al ajuste del

TABLA III

CARACTERIZACIÓN INTENSIDAD, EXPERIENCIA Y UTILIDAD EN EL USO DE INTERNET DEL MÉDICO EN UD (DIMENSIONES, PORCENTAJE DE MÉDICOS Y SIGNIFICACIÓN ESTADISTICA CHI CUADRADO)

\begin{tabular}{|c|c|c|c|c|}
\hline Dimensiones & $\begin{array}{l}\text { Intensidad, experiencia y } \\
\text { utilidad en el uso de internet }\end{array}$ & $\begin{array}{l}\text { Médicos en } \\
\text { UD }\end{array}$ & $\begin{array}{l}\text { Médico } \\
\text { tradicional }\end{array}$ & $\begin{array}{l}\text { Significación } \\
\text { estadística } p\end{array}$ \\
\hline \multicolumn{4}{|c|}{ Frecuencia de uso de internet desde su lugar de trabajo (Intensidad) } & 0,000 \\
\hline & Todos los días & 65,7 & 44,3 & \\
\hline & De 3 a 4 veces por semana & 19,2 & 22 & \\
\hline & De 1 a 2 veces por semana & 11,4 & 21 & \\
\hline \multicolumn{4}{|c|}{ Frecuencia de uso de internet fuera de su lugar de trabajo (Intensidad) } & 0,034 \\
\hline & Todos los días & 49,1 & 45,9 & \\
\hline & De 3 a 4 veces por semana & 67,7 & 55,6 & \\
\hline & De 1 a 2 veces por semana & 21,3 & 37,8 & \\
\hline \multicolumn{4}{|c|}{ Año de comienzo en el uso de internet para la práctica profesional (Experiencia) } & 0,000 \\
\hline & De 1995 a 2000 & 11,0 & 6,5 & \\
\hline & De 2000 a 2005 & 67,7 & 55,6 & \\
\hline & De 2005 a 2014 & 21,3 & 37,8 & \\
\hline \multicolumn{4}{|c|}{ Utilidad de internet para la práctica profesional (Utilidad) } & 0,000 \\
\hline & Muy Útil & 85,3 & 67,3 & \\
\hline & Útil & 14,2 & 29,4 & \\
\hline & Poco Útil & 0,5 & 2,8 & \\
\hline
\end{tabular}


TABLA IV

CONSECUENCIAS DEL USO DE INTERNET - MÉDICO EN UD (DIMENSIONES, PORCENTAJE DE MÉDICOS Y SIGNIFICACIÓN ESTADÍSTICA CHI CUADRADO)

\begin{tabular}{|c|c|c|c|c|}
\hline Dimensiones & $\begin{array}{l}\text { Intensidad, experiencia y utilidad en } \\
\text { el uso de internet }\end{array}$ & $\begin{array}{l}\text { Médicos } \\
\text { en UD }\end{array}$ & Médico tradicional & $\begin{array}{l}\text { Significación } \\
\text { estadística p }\end{array}$ \\
\hline \multicolumn{4}{|c|}{ Valoración de la información sobre dolor disponible en internet } & 0,008 \\
\hline & Muy relevante & 12,3 & 7,4 & \\
\hline & Relevante & 69,1 & 69,9 & \\
\hline & Poco relevante & 18 & 21 & \\
\hline \multicolumn{4}{|c|}{$\begin{array}{c}\text { Cree que consultar información sobre dolor en internet, mejora el conocimiento del } \\
\text { paciente y facilita su tratamiento }\end{array}$} & 0,000 \\
\hline & Mucho & 13,6 & 8,8 & \\
\hline & Algo & 47,4 & 43,0 & \\
\hline & Poco & 28,9 & 38,3 & \\
\hline & Nada & 10,1 & 9,8 & \\
\hline \multicolumn{4}{|c|}{ El uso de internet en su actividad profesional } & 0,000 \\
\hline & $\begin{array}{l}\text { Mejora la comunicación con otros } \\
\text { profesionales }\end{array}$ & 87,5 & 76,7 & \\
\hline & $\begin{array}{l}\text { Mejora la eficiencia y la productividad } \\
\text { de su trabajo }\end{array}$ & 83,6 & 78,2 & \\
\hline & Mejora la atención a los pacientes & 55,8 & 48,9 & \\
\hline & $\begin{array}{l}\text { Mejora la comunicación con los } \\
\text { pacientes }\end{array}$ & 35,9 & 20,3 & \\
\hline
\end{tabular}

TABLA V

VALORACIÓN DE LAS TECNOLOGÍAS DE LA INFORMACIÓN Y DE LA COMUNICACIÓN DEL MÉDICO EN UD (DIMENSIONES, PORCENTAJE DE MÉDICOS Y SIGNIFICACIÓN ESTADÍSTICA CHI CUADRADO)

\begin{tabular}{|c|c|c|c|c|}
\hline Dimensiones & $\begin{array}{l}\text { Intensidad, experiencia y } \\
\text { utilidad en el uso de internet }\end{array}$ & Médicos en UD & Médico tradicional & $\begin{array}{l}\text { Significación } \\
\text { estadística } p\end{array}$ \\
\hline \multicolumn{4}{|c|}{$\begin{array}{l}\text { La existencia de datos informatizados que permitan ver la evolución del estado clínico del } \\
\text { paciente es muy útil para la práctica clínica }\end{array}$} & 0,033 \\
\hline & Totalmente de acuerdo & 69,5 & 63,1 & \\
\hline & En parte de acuerdo & 28,3 & 35,1 & \\
\hline & En desacuerdo & 1,6 & 1,5 & \\
\hline \multicolumn{4}{|c|}{$\begin{array}{l}\text { Con el uso masivo de las tecnologías de la información y la comunicación existe un mayor } \\
\text { control de los errores }\end{array}$} & 0,002 \\
\hline & Totalmente de acuerdo & 22,3 & 16,7 & \\
\hline & En parte de acuerdo & 57,5 & 60,5 & \\
\hline & En desacuerdo & 19,2 & 22,8 & \\
\hline \multicolumn{4}{|c|}{$\begin{array}{l}\text { Mi práctica clínica me dice que es tan importante innovar en las cuestiones organizativas } \\
\text { de los servicios y la institución, como invertir en nuevas prácticas a través del uso de las } \\
\text { tecnologías de la información y comunicación }\end{array}$} & 0,000 \\
\hline & Totalmente de acuerdo & 68,2 & 54,5 & \\
\hline & En parte de acuerdo & 3408 & 43,0 & \\
\hline & En desacuerdo & 1,0 & 2,4 & \\
\hline
\end{tabular}


modelo, el R2 Nagelkerke es del 0,490. Se acepta la bondad de ajuste del modelo y las variables tiene poder explicativo conjuntamente (test de Hosmer-Lemeshow y Chi cuadrado respectivamente). La Tabla VI recoge los principales resultados de la estimación realizada.

De la estimación del modelo se desprende que todas las variables tienen un poder explicativo muy significativo sobre la adopción de un uso intensivo (médicos UD) de internet. Las relaciones de influencia son positivas, con excepción del factor relacionado con aquellos profesionales que están orientados hacia la información nacional. Así pues, se observa que los factores relacionadas con el uso de la información internacional; el uso de internet orientado hacia la información y la investigación y el uso de los sistemas de información orientados hacia fuera de la organización juegan un papel significativo en la adopción de un uso intensivo de internet. Ese mismo papel positivo lo juega el que los médicos realicen investigación; que consideren que el uso de internet en su actividad profesional mejora la comunicación con el paciente y que realicen sus actividades en centros públicos y en centros privados (titularidad mixta).

\section{DISCUSIÓN}

Los médicos que tratan el dolor como enfermedad se enfrentan a los beneficios y los riesgos que supone la introducción de las TIC, especialmente internet, en el ámbito de la salud (5-10). El acceso y el uso de estas nuevas tecnologías podría tener consecuencias, tanto sobre su práctica médica como sobre la configuración de dichas tecnologías, en el contexto de las organizaciones donde desarrollan su trabajo $(11,12)$ y su acceso a una fuente de información especializada que les permite una actualización constante de sus conocimientos (13-15).

Internet también puede ser configurado como una herramienta para la asistencia médica. Los profesionales médicos con labor asistencial, docente e investigadora pueden disponer de sistemas de información que le permitan construir y consultar el historial médico del paciente. Esta nueva gestión de la información clínica podría transformar tanto su práctica clínica como su manera de relacionarse con el paciente y con otros profesionales sanitarios. La escasa for-

TABLA VI

LOS DETERMINANTES DE ADOPCIÓN DE USOS INTENSIVOS DE INTERNET POR LOS

PROFESIONALES MÉDICOS (MÉTODO DE REGRESIÓN: ANÁLISIS LOGIA BINOMIAL; VARIABLE DEPENDIENTE: MÉDICO EN UD; VALOR 1, Sí, VALOR O, NO; COEFICIENTES ESTANDARIZADOS)

\begin{tabular}{|l|c|c|c|c|c|}
\hline & $\begin{array}{c}\text { Coeficiente } \\
\text { estimado }\end{array}$ & $\begin{array}{c}\text { Error } \\
\text { estándar }\end{array}$ & Wald & $\begin{array}{c}\text { Significación } \\
\text { estadística } p\end{array}$ & Exp (B) \\
\hline $\begin{array}{l}\text { Orientado hacia la información } \\
\text { internacional }\end{array}$ & 1,511 & 0,122 & 154,148 & 0,000 & 4,532 \\
\hline $\begin{array}{l}\text { Internet orientado hacia la } \\
\text { información investigación }\end{array}$ & 0,953 & 0,086 & 122,001 & 0,000 & 2,595 \\
\hline $\begin{array}{l}\text { Orientado hacia la información } \\
\text { nacional }\end{array}$ & $-0,296$ & 0,087 & 11,545 & 0,001 & 0,744 \\
\hline $\begin{array}{l}\text { Sistemas de información } \\
\text { orientados hacia fuera de la } \\
\text { organización }\end{array}$ & 0,267 & 0,085 & 9,806 & 0,002 & 1,306 \\
\hline $\begin{array}{l}\text { Realiza en su actividad profesional } \\
\text { investigación }\end{array}$ & 0,655 & 0,203 & 10,418 & 0,001 & 1,925 \\
\hline $\begin{array}{l}\text { El uso de internet en su } \\
\text { actividad professional mejora la } \\
\text { comunicación con los pacientes }\end{array}$ & 1095 & 0,179 & 37,242 & 0,000 & 2,990 \\
\hline $\begin{array}{l}\text { Titularidad del centro (público o } \\
\text { privado - mixto) }\end{array}$ & 0,411 & 0,185 & 4,934 & 0,026 & 1,509 \\
\hline & & & & -2 Log-likelihood & 951,749 \\
\hline & & & R2 Cox-Snell & 0,363 \\
\hline & & & $\begin{array}{l}\text { R2 Nagelkerke } \\
\text { Prueba de } \\
\text { Hosmer- }\end{array}$ & 0,490 \\
\hline & & & & & \\
\hline
\end{tabular}


mación de los profesionales médicos en el uso de las Tecnologías de la Información y la Comunicación y las barreras asociadas con la falta de confidencialidad y seguridad de los datos pueden suponer un problema a la hora de utilizar este tipo de recursos (16). Además, estos sistemas de información también podrían tener repercusiones sobre el control o la autonomía de los profesionales en su práctica diaria, lo que podría tener un impacto en la calidad, en la eficiencia y en los costes de su institución.

Las consecuencias reales en cualquier sector de actividad de la introducción y uso de las tecnologías sobre la cualificación, las actividades, la organización y los resultados del trabajo dependen tanto de las características formativas, de las habilidades de aprendizaje, de la experiencia y de la interacción de las organizaciones con su entorno, como del patrón de competitividad económico y social. En este sentido, durante la introducción de un proceso de innovación tecnológico se establece un complejo tejido de interacciones organizativas, productivas, laborales, institucionales y culturales que condicionan el propio proceso en función de la dirección de estas interrelaciones. Por tanto, el impacto de las tecnologías solo se puede comprender a partir del análisis de estas complejas interacciones dentro del sistema social en el que se producen $(17,15)$. La identificación, caracterización y determinación del uso intensivo de internet nos muestra que los médicos encuestados no son ajenos a esta realidad.

El uso de las Tecnologías de la Información y la Comunicación e internet en la práctica profesional pone de relieve la importancia de la interacción de las variables relacionadas con el contexto organizativo de uso de estas tecnologías $(18,19)$.

En general, en los hospitales es donde se utilizan estas tecnologías principalmente, ya que son el tipo de instituciones que pueden tener la capacidad de inversión necesaria. Además, en muchas ocasiones las estructuras jerárquicas de estos centros dotan de un gran poder a los profesionales de más prestigio, lo que facilita su autonomía a la hora de decidir qué tipo de tecnologías se utilizan en sus servicios.

En la valoración de los usos de internet por parte de los profesionales destaca la idea de que la introducción y uso de este tipo de tecnologías no pueden abordarse desde una perspectiva determinista. Es decir, los profesionales son conscientes que en estos procesos no se produce una adaptación de sus conductas automáticamente, sino que se producen interacciones entre factores humanos, culturales, organizativos y tecnológicos que moldean tanto su conducta como la propia tecnología.

En general, los profesionales médicos de unidades de dolor tienen una percepción positiva del uso de las Tecnologías de la Información y la Comunicación e internet en su actividad profesional.

Esta visión queda matizada fundamentalmente por dos cuestiones:

En primer lugar, por el uso que los pacientes pueden hacer de estas tecnologías y por las consecuencias que de este uso puedan derivarse sobre la relación médicopaciente y sobre la salud del propio paciente.

En segundo lugar, esta visión positiva se ha de enfrentar a las complejas interacciones que suponen la introducción y uso de estas tecnologías en los centros donde desarrollan su actividad, ya que esto viene acompañado de una reestructuración social y organizativa que afecta directamente a sus actividades cotidianas.

En el caso de los profesionales encuestados, las variables que más peso tienen sobre este impacto no son las relacionadas con las características de los individuos (formación, habilidades de aprendizaje y experiencia) sino aquellas variables que tienen relación con sus lugares de trabajo con la relación de los centros proveedores de servicios con su entorno y con el patrón de competitividad económico y social de estos.

Esto se pone de manifiesto tanto en la identificación y caracterización del médico en unidades de dolor como en sus determinantes.

\section{CONCLUSIONES}

Se observa que existe un porcentaje mayor de utilización de TIC e internet en médicos de unidades de dolor que trabajan en un hospital, que trabajan en más de un centro asistencial cuya titularidad es mixta (públicaprivada), que realizan más actividades que la práctica asistencial, que tienen más años de experiencia en el uso de internet y encuentran esta tecnología más útil, que valoran más positivamente la información disponible en internet y dialogan con los pacientes sobre esta fuente de información.

Además, también existe un porcentaje mayor de médicos en unidades de dolor que valoran más positivamente las consecuencias de esta tecnología sobre la mejora de la relación y la comunicación con sus pacientes, la eficiencia y productividad de su trabajo.

Finalmente, el porcentaje más elevado corresponde a un nuevo perfil profesional que son aquellos médicos que hacen un uso intensivo de los sistemas de información y valoran más positivamente el impacto de las tecnologías de la información y la comunicación en su práctica clínica.

\section{AGRADECIMIENTOS}

A los alumnos del Máster del dolor de la USAL por suministrarnos los datos para el estudio.

\section{CONFLICTO DE INTERESES}

En ningún caso la financiación del proyecto ha supuesto un condicionante o limitación a la total independencia de la investigación.

\section{BIBLIOGRAFÍA}

1. Carrillo Castrillo JA. Caracterización de la accidentalidad laboral en el sector industrial andaluz en el periodo 20032008. Aplicaciones en el diseño y evaluación de programas de intervención (Tesis). Sevilla: Universidad de Sevilla; 2014.

2. OECD. Information Technology Outlook. OCDE. [Fecha de consulta: 25 de septiembre de 2018]. Disponible en: http:// www.oecd.org/dataoecd/20/47/33951035.pdf. 
3. Anderson GF, Frogner BK, Johns RA, Reinhardt UE. Health care spending and use of information technology in oecd countries. Health Aff. 2006;25(3):819-31. DOl: 10.1377/ hlthaff.25.3.819.

4. Burrows R, Nettleton S, Pleace N. Virtual community care? Social policy and the mergence of computer mediated social support. Information, Communication \& Society 2000;3(1):95-121. DOl: 10.1080/136911800359446.

5. Kassirer JP. Patients, physicians, and the internet. Health Aff. 2000;19(6):115-23. DOI: 10.1377/hlthaff.19.6.115.

6. Miller TE, Derse AR. Between strangers: the practice of medicine online. Health Aff. 2002;21(4):168-79. DOI: 10.1377/hlthaff.21.4.168.

7. Levy J, Strombeck R. Health benefits and risk of the internet. J Med Syst. 2002;26(6):495-510.

8. Lorenzo S, Mira JJ. Are Spanish physicians ready to take advantage of the Internet? World World Hosp Health Serv. 2004;40(3):31-5.

9. Hasty RT, Garbalosa RC, Barbato VA, Valdes PJ Jr, Powers DW, Hernandez E, et al. Wikipedia vs peer-reviewed medical literature for information about the 10 most costly medical conditions. J Am Osteopath Assoc. 2014;114(5);368-73. DOI: 10.7556/jaoa.2014.035.

10. Love T, Laier C, Brand M, Hatch L, Hajela R. Neuroscience of Internet Addiction: A Review and Update. Behav Sci. 2015;5(3):388-433. DOl: 10.3390/bs5030388.

11. Heath C, Luff P, Sanchez M. Technology and medical practice. Sociol Health IIIn. 2003;25:75-96.

12. Muriel J, Sánchez Ledesma MJ, López Millan JM, GarcíaCenador MB. Study of the uses of Information and Communication Technologies by Pain Treatment Unit Physicians. J Med Syst 2017;41(5):78. DOI: 10.1007/s10916-017-0726-3.
13. Chew F, Grant W, Totes R. Doctors on-line: using diffusion of innovations theory to understand internet use. Fam Med. 2004;36(9):645-50.

14. Dear BF, Gandy M, Karin E, Staples LG, Johnston L, Fogliati VJ, et al. The Pain Course: a randomised controlled trial examining an internet-delivered pain management program when provided with different levels of clinician support. Pain. 2015;156(10):1920-35. DOI: 10.1097/j. pain. 0000000000000251 .

15. Muriel J, García-Cenador MB, López Millan JM, Juanes JA, Sánchez-Ledesma MJ. Use of Information and Communication Technologies in Clinical Practice Related to the Treatment of Pain. Influence on the Professional Activity and the Doctor-Patient Relationship. J Med Syst. 2017;41(5):77. DOI: 10.1007/s10916-0170724-5.

16. Cullen RJ. In search of evidence: family practicionaers' use of Internet for clinical information. J Med Library Association 2002;90(4):370-9

17. Wilkinson JF, Short HL, Wilkinson S, Mander A. Commissioning for menopause specialist services: A local perspective: An internet-based survey to assess the potential demand for menopause care in West Cheshire and the skills of local primary care clinicians in this field, with a view to informing future commissioning locally. Post Reprod Health. 2015;21(3):98104. DOI: 10.1177/2053369115595309.

18. Walsh $\mathrm{K}$ Neuroscience education on the internet: the next steps. Malays J Med Sci. 2014;21(6):75.

19. De Lima Verde Brito TD, Baptista RS, de Lima Lopes PR, Haddad AE, Messina LA, Torres Pisa I. The Collaborative Coordination of Special Interest Groups on the Telemedicine University Network (RUTE) in Brazil. Stud Health Technol Inform. 2015;21(4):1010-16. 